\title{
Wireless innovation forum best papers journal issue: our overview
}

\author{
Bruce Fette $^{1} \cdot$ Jeffrey H. Reed ${ }^{2}$
}

Published online: 18 February 2017

(c) Springer Science+Business Media New York 2017

It is truly a pleasure to be able to introduce this collection of excellent journal papers selected from the best papers presented at the Wireless Innovation Conference held in March 2016 in Reston Virginia. The best papers were selected based on five criteria including substantial advancement to the art and greatest impact to industry. These best papers were then invited to be expanded into full journal articles for this edition of the Springer Journal.

We have sorted the journal article submissions into three topic areas:

Cognitive Technology, Spectrum Management, and Spectrum Efficiency (6 papers)

Waveforms and Network Protocols (5 papers)

Receiver Design of Software Defined Radios (3 papers)

In the area of Cognitive Technology, Spectrum and Spectrum Efficiency, Asadi et al. teach means to make use of Meta-Cognition, selection from multiple cognitive engines (CE) to best understand and respond to its environment in both speed (computation), accuracy and ability to deliver communication successfully for a given environmental spectrum activity. Yrjola et al. provide an excellent overview of stakeholder viewpoints and business opportunities in the newly defined Citizens Broadband Radio Service (CBRS) and elements that can help insure the success of this new service. Sohul et al. address a collaborative spectrum sharing auction approach for use by

Bruce Fette

bfette@ida.org

Jeffrey H. Reed

reedjh@vt.edu

1 Institute for Defense Analyses, Alexandria, VA, USA

2 Virginia Tech, Blacksburg, VA, USA primary and secondary users that make use of Hidden Markov Models to estimate spectrum auction prices that assures spectrum availability, efficiency, cost objectives and quality of service to users. Bastidas et al. develop techniques for Wideband Distributed Spectrum Sharing with Immediate Multiple Access. A channelized radio architecture is proposed and implemented for distributed spectrum sharing that includes spectrum access system, wideband sensing, sophisticated fast rendezvous based on OFDM, and a sophisticated CSMA time slotted MAC protocol. Hirzallah et al. explore methods to enable spectrum sharing between radar and LTE via use of steepest decent or non-steepest decent waveform pre-coders to reduce interference from radar systems. Cai et al. demonstrates the ability to model spectrum usage of a transmitter and associated beam pattern properties of the antennas and corresponding propagation losses in order to be able to predict cross interference between various spectrum users, and thereby achieve greater spectrum allocation efficiency.

In the area of Waveforms and Protocols, Peken et al. explore methods for channel estimation and effects interference from adjacent cells on pilot contamination via blind channel (ICA, SVD, EVD), semi-blind (SVD), ICA based blind channel, and ICA adaptive blind channel techniques. Abello et al. address the spectral efficiency and complexity issues in waveform design for very fast data rates using Faster Than Nyquist (FTN) Modulation techniques, and thus advances the art in spectrum efficiency at relatively low Eb/N0. Ngassa et al. have two complementary papers on security and privacy. In the first paper, her team addresses secret key generation based on local channel conditions including addressing security and privacy with key entropy up to 127 bits. In the second paper her team addresses how information with high redundancy such as imagery and video can be denied to eaves-dropping via 
beam pattern management that includes transmitting noise toward the eavesdropper while sending the intended receiver the intended information on a separate beam. Vanhoy et al. explore detection and characterization of radar signal properties showing advanced signal processing combined with support vector machines to create high accuracy of signal recognition.

In the area of receiver design, Dr. Fred Harris and his team introduce flexible channelizer receiver design techniques that can cope with receiving multiple OFDM signals from multiple sources that are not frequency or time aligned and having different bandwidths such as will happen in LTE base stations. Chaudhari et al. report on how to manage complexity of an iterative receiver regarding channel estimation, including how to do timing, frequency and phase offsets inside the iterative loop, and how to do the log likelihood ratios for LDPC parity bits. Ribeiro et al. discuss rapid prototyping of a high data rate 5G LTE features and OFDM waveforms via Analog Devices front end development board and XILINX backend development board.

We are certain that this journal issue will provide value both to immediate equipment development and the deployment of $5 \mathrm{G}$ systems with fundamental new techniques that will substantially impact designs beyond $5 \mathrm{G}$. We hope you enjoy reading these breakthroughs in techniques in wireless innovation!

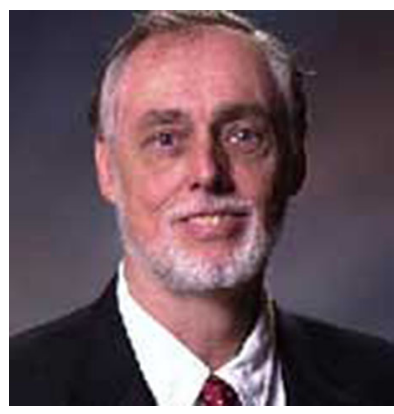

Bruce Fette Ph.D. is an Adjunct Research Staff Member with Institute for Defense Analyses. His work at IDA involves performance analyses, requirements analyses, new architecture analyses and design recommendations regarding various communication architectures. Previously Dr. Fette was with DARPA, where he created the Communications in Extreme RF Spectrum Environments (CommEx) Program, and also managed the Advanced Wireless Networked Systems (AWNS), Wireless Network After Next (WNaN), Mobile Networking MIMO (MnM), Labrador, Remote Detection of Activity (RDA), Disruption Tolerant Networking
(DTN), Connectionless Networks (CN) and five SBIR programs in the communication technology area. Prior to DARPA, Dr. Fette was Chief Scientist, Dan Nobel Fellow and Science advisory Board member at General Dynamics and Motorola, where he lead work in secure voice communication and various software defined radio programs including Speakeasy I and II, Digital Modular Radio (DMR), JASORS and ACN. His early career at Motorola involved development of MOS LSI including the first eight bit microprocessor, CAD cells and CAD tools for LSI design, and DSP LSI processors for LPC speech analysis and synthesis. Dr. Fette was also a founding member of the SDR Forum, now known as the Wireless Innovation Forum, and served as the technical committee chair. He is also a lifetime member of IEEE. Dr. Fette has 36 patents, 39 papers, 4 books, and has received the 2009 SDR Forum Presidents Award, the 2011 Winn Forum Fellow award, and the 2014 Secretary of Defense Medal for Exceptional Public Service award.

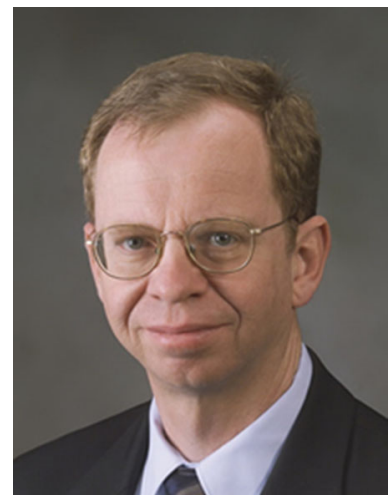

Jeffery H. Reed is the Willis G. Worcester Professor in the Bradley Department of Electrical and Computer Engineering at Virginia Tech. He currently serves as Founding Director of Wireless@Virginia Tech, one of the largest and most comprehensive university wireless research groups in the US which he founded in 2006 and served as its first director. In 2010, he founded the Ted and Karyn Hume Center for National Security and Technology and served as its Interim Director. Dr. Reed is co-founder of Cognitive Radio Technologies (CRT), a company that is commercializing of the cognitive radio technologies produced for military applications; Federated Wireless, a company that is developing spectrum sharing technologies; and for PFP Cybersecurity, a company that specializes in security for embedded systems. He has also served as a consultant for approximately thirty organizations, covering topics such as merger evaluation, network neutrality, and band planning. Dr. Reed served on the President's Council of Advisor in Science and Technology (PCAST) Advisory Group on how to transition federal spectrum for commercial economic benefits. In 2014, Dr. Reed was selected to be a member of CSMAC, the advisory group on spectrum issues for the US Department of Commerce. In 2014, Dr. Reed served as co-general chair for the IEEE Dynamic Spectrum Access Network (DySPAN) conference. Dr. Reed is a Fellow of the IEEE for contributions to software radio and communications signal processing and for leadership in engineering education. $\mathrm{He}$ is a past recipient of the College of Engineering Award for Excellence in Research. Dr. Reed received his BS, MS, and Ph.D. degrees from the University of California, Davis. 